\title{
Reflets
}

Revue ontaroise d'intervention sociale et communautaire

\section{Le développement économique communautaire : les défis des femmes du Nord-Ouest}

\section{Paula Haapanen}

Volume 5, numéro 1, printemps 1999

Pratiques et développement économique communautaire

URI : https://id.erudit.org/iderudit/026252ar

DOI : https://doi.org/10.7202/026252ar

Aller au sommaire du numéro

Éditeur(s)

Reflets : Revue ontaroise d'intervention sociale et communautaire

ISSN

1203-4576 (imprimé)

1712-8498 (numérique)

Découvrir la revue

Citer cet article

Haapanen, P. (1999). Le développement économique communautaire : les défis des femmes du Nord-Ouest. Reflets, 5(1), 116-122.

https://doi.org/10.7202/026252ar

Tous droits réservés (C) Reflets : Revue ontaroise d'intervention sociale et communautaire, 1999
Ce document est protégé par la loi sur le droit d'auteur. L'utilisation des services d'Érudit (y compris la reproduction) est assujettie à sa politique d'utilisation que vous pouvez consulter en ligne.

https://apropos.erudit.org/fr/usagers/politique-dutilisation/ 


\title{
Le développement économique communautaire: les défis des femmes du Nord-Ouest
}

\author{
Paula Haapanen \\ Adjointe à la coordination du développement économique \\ Centre des femmes francophones du Nord-Ouest de l'Ontario
}

Au Centre (autrefois Comité) des femmes francophones du NordOuest de l'Ontario (CFFNOO), nous travaillons depuis 1995 sur le dossier du développement économique. Précisons que la raison d'être du Centre est de lutter contre la violence faite aux femmes et l'agression à caractère sexuel ainsi que d'offrir des services de soutien et d'intervention en français. Nous avons décidé de poursuivre le dossier du développement économique après avoir constaté que les femmes abusées qui quittent leur conjoint ou leur conjointe se retrouvent presque sans ressources, tant psychologiques que financières. Quand elle ne peuvent subvenir seules à leurs besoins et à ceux de leur famille, elles se voient dans l'obligation de recourir à l'assistance sociale ou à d'autres formes d'aide. Notre but est de lutter contre de tels problèmes et d'améliorer ainsi la situation de toute femme francophone.

Plus nous avancions dans notre recherche sur les besoins des femmes ayant un vécu de violence, plus nous nous rendions compte que toute femme pouvait bénéficier de notre travail et de nos ressources. C'est alors que notre optique a changé pour inclure toute femme francophone de la région du Nord-Ouest de l'Ontario.

Quelle est la situation économique actuelle des femmes francophones du Nord-Ouest ontarien ? Les statistiques sont très révélatrices. Dans cette région, nous notons une différence marquée dans la distribution des salaires entre les hommes et les 
femmes en général. Selon le recensement de 1991, plus de deux tiers des femmes $(69,9 \%)$ ont un salaire de moins de $20000 \$$ par année; 35,6\% des hommes, soit environ le tiers, font partie de cette catégorie. Par contre, presque la moitié des hommes dans le Nord-Ouest de l'Ontario (49\%) gagnent plus de 30000 \$ par année tandis que chez les femmes, seulement $14,9 \%$ ont reçu plus de $30000 \$$. On constate donc que le salaire moyen des hommes est deux fois plus élevé que celui des femmes (Cormier :1).

Une différence plus marquée émerge de l'analyse de la situation dans certaines municipalités. Par exemple, la municipalité de Manitouwadge, située à $400 \mathrm{~km}$ au nord-est de Thunder Bay, publie un profil pour ses nouveaux arrivants et pour les investisseurs potentiels. On y inclut diverses statistiques dont celles-ci : en 1995, le salaire moyen pour un homme était de 52800 \$; il était de $10900 \$$ pour les femmes (Statistique Canada : table 2). Autrement dit, ces dernières reçoivent moins du quart du salaire des hommes. Comment faire vivre une famille avec 10900 \$ par année? De plus, ces statistiques n'isolent pas la population francophone. Et ici aussi, la différence est importante. La situation est grave pour toutes les femmes du Nord-Ouest de l'Ontario mais elle l'est davantage pour les francophones.

Dans cet article, je soulèverai certains des problèmes et défis de la femme francophone de la région Nord-Ouest pour ensuite présenter les solutions que le Centre des Femmes Francophones se propose de mettre en place.

\section{Problèmes}

Le problème le plus criant est que les femmes francophones sont systématiquement oubliées dans la planification des programmes de développement économique dans la région, provoquant chez elles un manque de confiance en un système gouvernemental et social qui est censé les aider. Les femmes francophones ont le goût de travailler, de démarrer des micro-entreprises, de gagner 
leur pain et de vivre plutôt que de vivoter. Pourtant, quand elles doivent faire face à un système politique et à des attitudes sociétales qui ne reconnaissent pas leurs besoins réels, elles sont démoralisées et n'ont plus le courage de poursuivre leurs initiatives. Beaucoup de femmes ont déjà fait l'exercice de remplir les formulaires, les questionnaires, de faire des appels pour se renseigner sur un emploi - souvent en anglais faute de personnel qualifié parlant français - et elles se retrouvent plus souvent qu'autrement à la case départ. À la fin, elles abandonnent, leur effort n'aboutissant à rien.

Comment cette ignorance ou ce désintérêt systémique se manifestent-t-ils? D'abord, il y a peu de services en français dans le domaine du développement économique, même dans les huit régions désignées dans le Nord-Ouest par la Loi sur les services en français. Les sociétés d'aide au développement des collectivités du nord de l'Ontario (SADC) dans le Nord-Ouest n'ont pas toujours du personnel qui parle français et qui est, en même temps, qualifié pour prodiguer les conseils pertinents sur les prêts ou les programmes disponibles du développement économique. Au niveau de la formation, les cours qui sont disponibles et abordables financièrement en français sont très peu nombreux. Qui plus est, il manque énormément de ressources en français, présentées dans un langage simple et clair, qui traitent de tout ce qui concerne le développement économique. Des questions telles que comment faire un plan d'affaires, où trouver les numéros de téléphone des agences qui offrent les services en français, comment faire la tenue des livres à la maison, ne trouvent pas réponse. De plus, les ressources qui existent ne sont pas centrées sur les besoins de la population du Nord-Ouest de l'Ontario et ne reflètent pas la réalité de la région. Souvent on utilise des données qui viennent du Sud-Est ou du Nord-Est, là où il y a une plus grande concentration de francophones et où la conjoncture est en conséquence très différente. Il n'y a pas assez de documentation sur les démarches déjà entreprises dans le Nord-Ouest, sur les problèmes et obstacles déjà vécus et surmontés et sur les solutions possibles dans la programmation pour la région. Enfin, on n'a même pas tenu compte des problèmes courants, quotidiens, 
auxquels on fait face. Par exemple, quand on trouve un emploi, on a peut-être besoin de gardiennes pour les enfants ou les personnes âgées à la maison. Est-ce que ces services sont disponibles dans la municipalité ou encore, le sont-ils en français? Si on a besoin de se déplacer pour l'emploi ou pour une formation et qu'on n'a pas de voiture, quels sont les moyens de déplacement disponibles et qui va les payer, surtout si on doit voyager entre 300 à $500 \mathrm{~km}$ pour de la formation ?Voici quelques exemples qui démontrent le peu d'importance donné aux femmes francophones comme participantes essentielles à l'économie. Voici quelquesuns des obstacles contre lesquels nous luttons continuellement pour améliorer la situation des femmes francophones du NordOuest de l'Ontario.

\section{Solutions}

Comment répondre aux problèmes mentionnés ci-dessus? Le CFFNOO a effectué, en septembre 1998, une étude qui indique qu'on doit «considérer les options dites non-traditionnelles afin d'inciter les femmes francophones à contribuer à l'économie et engendrer un développement et une croissance économique ainsi qu'une création d'emploi basés sur les besoins de la communauté et non déterminés par les banques et les grandes entreprises» (CFFNOO: 1). Notre rôle au Centre par rapport au développement économique est d'aider les femmes à créer des emplois stables, avec des salaires suffisants pour vivre décemment, en leur fournissant tout l'appui technique et moral possible.

Plusieurs moyens considérés comme non-traditionnels ont été explorés dans le contexte de notre projet. Qu'appelle-t-on un moyen non-traditionnel? L'entrepreneuriat ou les microentreprises, les caisses d'entraide - autrement connus comme les cercles d'emprunt - et les coopératives font partie de cette catégorie. Il n'y a peut-être rien de nouveau ou de nontraditionnel dans ces concepts. Ils existent depuis longtemps. La seule chose ayant changé est que ceux-ci font maintenant partie 
de la pensée courante du monde des affaires comme étant des choix viables pour les femmes. Le climat est certes favorable à ces démarches mais les femmes ont enfin dit qu'elles en ont marre de ne rien trouver pour elles-mêmes et elles n'ont pas envie d'aller travailler pour les autres non plus. Elles veulent mieux contrôler leur destin, c'est-à-dire travailler à leur propre compte, quand elles le veulent et où elles le veulent.

Or, les femmes - comme les hommes - ne sont pas toutes faites pour l'entrepreneuriat dans son contexte traditionnel. Une définition plus englobante de ce concept permet de voir comment il peut offrir une niche pour tout le monde. Il ne s'agit plus forcément d'une personne qui essaie de bâtir à elle seule une entreprise. Par exemple, tout membre d'une coopérative est aussi un entrepreneur; au lieu de travailler tout seul, nous travaillons en équipe pour réaliser des projets collectifs. Une équipe veut dire un réseau d'entraide, des conseils, des suggestions et un coup de main quand il le faut. Personne ne joue un rôle moindre; les rôles sont tout simplement différents. Une telle concertation renforce l'idée de développement communautaire et amoindrit la chance d'échec tout en respectant l'idée de l'entrepreneuriat, mais vue dans une autre optique.

Au Centre des femmes francophones du Nord-Ouest de l'Ontario, nous privilégions cette optique élargie. Nous essayons de promouvoir l'idée d'entraide et de solidarité entre les femmes francophones à travers des séances de réseautage où elles peuvent se réunir non seulement pour échanger leurs cartes d'affaires mais aussi pour partager leurs connaissances, découvrir des ressources qui leur sont disponibles et éventuellement créer des partenariats entre elles. De plus, nous nous proposons la publication d'un bulletin qui s'intitule Femmes vers l'entrepreneuriat, dans lequel nous retrouverons des articles sur le développement économique communautaire et d'autres sujets pertinents tels que l'exploitation maximale d'un plan d'affaires, ou la recherche de ressources financières. Nous trouverons également des nouveaux services et produits dans le "coin commercial», une rubrique de publicité gratuite où les femmes pourront annoncer leurs entreprises. Enfin, le bulletin comprendra aussi une rubrique 
permettant d'afficher toutes les activités communautaires et régionales de développement économique. Femmes vers l'entrepreneuriat se veut un outil de réseautage efficace, en français, pour les femmes francophones, par les femmes francophones.

Nous considérons les moyens de développement économique communautaire non-traditionnels que nous développons comme des incubateurs dans le sens où nous partons d'une idée d'entreprise ou d'une entreprise déjà existante et visons à la développer jusqu'à ce qu'elle puisse subsister d'elle-même. De la conception d'un projet à sa réalisation, nous fournissons pour un coût minime les bureaux, l'équipement, la formation nécessaire et les conseillères, tout ceci dans le but de créer une ambiance propice à la réussite. En moyenne, les incubateurs encadrent les projets pour un minimum de deux ans parce que le processus est long et qu'on avance à petits pas. Notre but est de créer de la place pour au moins dix entreprises, mais cela se fera en fonction du nombre de femmes qui auront recours à nos services. Plusieurs modèles peuvent être utilisés pour l'organisation de ces incubateurs, mais on n'a pas encore choisi celui qu'on privilégiera. La décision sera prise suite à notre analyse de besoins.

Pour conclure, il faut parler de notre plus bel outil - la promotion - qui se fait de bouche à oreille. Le réseautage constitue le moyen le plus efficace pour faire affaire dans notre communauté. Si on a besoin d'un service ou d'un produit et qu'on sait qu'il existe chez nous, il vaut mieux privilégier le fournisseur local, surtout dans le cas des femmes. Nous savons tous que c'est peut-être moins cher chez les grandes entreprises, mais l'argent quitte alors notre communauté. Souvent, une femme qui vient juste de créer sa propre entreprise n'a pas les moyens de concurrencer les grandes sociétés, au niveau des campagnes de publicité ou des soldes. Mais acheter localement signifie que l'argent qu'on dépense pour les biens et services restera davantage dans la communauté ou autrement dit, l'argent est réinvesti parmi les personnes qui l'ont dépensé. Le principe est simple et toute la communauté en bénéficie. 


\section{L'avenir?}

Nous commençons déjà à réaliser quelques résultats concrets. Le chemin paraît long mais non pas désespéré.Après avoir fait toutes les études et cherché toute l'information pertinente, nous sommes maintenant prêtes à fournir les services de qualité aux femmes francophones de la région. On veut créer quelque chose de durable, facilitant l'épanouissement de notre communauté et aidant les femmes francophones à devenir financièrement autonomes pour qu'elles puissent profiter complètement de leur potentiel personnel et professionnel.

\section{Bibliographie}

CENTRE DES FEMMES FRANCOPHONES DU NORD-OUEST DE L'ONTARIO (CFFNOO) (1997). Synthèse du projet de recherche sur la contribution économique des femmes francophones du Nord-Ouest de l'Ontario, Thunder Bay, Ontario, CFFNOO.

CORMIER, Charline (septembre 1996). Projet de recherche sur la contributuion économique des femmes francophones du Nord-Ouest de l'Ontario, Thunder Bay, Ontario, CFFNOO.

STATISTICS CANADA (septembre 1997). Community Profile, Manitouwadge Economic Development Corporation, Manitouwadge, Ontario, appendice 1, table 2. 